\title{
GIANGIORGIO TRISSINO: INNOVADOR, POETA Y MÁXIMO TEÓRICO DE LA MÉTRICA ITALIANA RENACENTISTA
}

\author{
ISABel PARAíso
}

\begin{abstract}
Resumen: Este trabajo presenta los aportes e innovaciones de Giangiorgio Trissino (1478-1550) en el campo de la Métrica italiana. Con sus obras Sophonisba e Italia liberata da' Goti consagró la utilización del verso suelto para obras extensas, buscando naturalidad y reproducir la grandeza del hexámetro. Y su Poética contiene la teoría métrica italiana más completa, sistemática y sensible escrita hasta entonces. De ahí su carácter modélico.
\end{abstract}

Palabras-clave: Métrica italiana, Trissino, verso suelto, pies, metros, poemas.

\begin{abstract}
This paper presents Giangiorgio Trissino's (1478-1550) contributions to and innovations in the field of Italian metrics. After the publication of his Sophonisba and Italia liberata da' Goti blank verse came into widespread use for lengthy works as a means of imitating the grandeur of the hexameter without sounding pretentious. His Poetica contains the most complete, systematic and sensitive theory of Italian meter written up to that time. This made him a model to be followed.
\end{abstract}

Key-words: Italian versification system, Trissino, blank verse, metric feet, meter in poetry, poems. 



\section{INTRODUCCIÓN}

Trissino, il più sottile ingegno metrico del

Rinascimento

(G. GORNI).

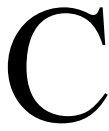

ADA literatura en lengua moderna cuenta con un fundador de su canon métrico. Con alguien que, sintiendo la poesía y conociéndola en profundidad, se aplica a desentrañar sus constituyentes verbales y sus esquemas; alguien que configura la teoría métrica. Eso suele suceder cuando la lengua nacional ha adquirido ya una madurez notable, y cuando la literatura nacional cuenta con valiosos modelos dignos de imitación. En la literatura española, ese papel corresponde al jesuita Juan Díaz Rengifo (Arte Poética Española, 1592) ${ }^{1}$. $\mathrm{Y}$ en la literatura italiana, a Messer Giangiorgio Trissino ( $L a$ Poética, 1529 y 1563).

Este trabajo aspira a presentar las teorías métricas del polígrafo humanista Giangiorgio Trissino (o Gian Giorgio, o Giovan Giorgio, o Giovanni Giorgio, pues de todos estos modos es citado). Porque, efectivamente, en la historia de la Métrica italiana (que, como es sabido, serviría de modelo para toda Europa, y muy en particular para España), descuellan tres nombres: Dante, el fundador; Antonio da Tempo, el primer expositor; y Giangiorgio Trissino, el configurador del canon,

1 Cfr. PARAÍSO, Isabel: "Fundación del canon métrico: El Arte Poética Española, de Juan Díaz Rengifo", en Isabel Paraíso (coordinadora): Retóricas y Poéticas Españolas, siglos XVI-XIX. Valladolid: Universidad de Valladolid, 2000, pp. 47-93. 
el creador de todo un sistema de análisis ${ }^{2}$.

Por otra parte, el vínculo entre Trissino y sus predecesores teóricos es manifiesto. Por Dante siente profundísima admiración: conoce muy bien toda su obra, y ejemplifica con ella siempre que puede. Fue Trissino quien tradujo del latín y publicó por primera vez (1529) el pequeño e incompleto pero espléndido texto De Vulgari Eloquentia de Dante (escrito probablemente hacia 1304). La sintonía entre ambos autores es tal, que muchos contemporáneos creyeron que el tratadito era de Trissino, y que lo había publicado bajo el nombre de Dante.

En cuanto a Antonio da Tempo (o "de Tempo", como Trissino escribe, y también Rengifo), su Summa Artis Rithimici Vulgaris Dictaminis era la única guía existente sobre los tipos de poemas en italiano, dada la no circulación del tratado de Dante hasta que Trissino lo exhumó. El mismo Tempo se consideraba innovador absoluto en esta materia, como afirma en su libro. Y durante dos siglos, Tempo fue considerado el único maestro en el campo de la versificación. Trissino en varios pasajes lo tiene en cuenta, y recoge sus ideas siempre que le parezcan válidas para describir el estado actual de la poesía italiana, que es su objetivo. No obstante, dada la gran diferencia de años entre la aparición de la Summa Artis (1332) y la primera parte de la Poetica de Trissino (1529), casi dos siglos, y dada la revolución tranquila pero profunda

También cabría mencionar a un influyente coetáneo de TRISSINO, Pietro BEMBO, cuyas Prose della Volgar Lingua aparecieron en 1525, pero cuyo sistema métrico - que en su obra ocupa un papel muy secundario-, muchísimo menos desarrollado que el de TRISSINO (1529), nuestro autor no lo tiene en cuenta. Bembo divide los poemas italianos en tres categorías: formas regulares (con estructura fija: sextina, terceto, octava), formas libres (con estructura variable, como el madrigal), y formas "mezcladas" (con estructura en parte libre y en parte variable: soneto, balada, canción).

Entre TEMPO y TRISSINO, podríamos recordar los tratados de GIDINO DI SOMMACAMPAGNA (Tractato et la arte delli rithimi vulgari, c. 1381-84) y de Francesco BARATELLA (Compendio ritimale, 1447), que son sólo versiones descafeinadas del de TEMPO y con pequeñas adiciones. El brevísimo De Componendis in lingua italiana Versibus Compendiolum (c. 1497) de Guido STELLA tampoco influyó en sus contemporáneos. Fuera de la órbita de TEMPO se sitúa el comentario a las Rimas de PETRARCA (1478) por Francesco PATRIZZI, obra encargada por el Duque de Calabria, Alfonso de Aragón. 
que Petrarca había introducido en la poesía italiana (Canzoniere, escrito entre el 1327 y el 1374), era normal que una parte de la Summa Artis ya no fuera válida, porque no reflejaba el panorama poético que Trissino tiene delante.

\section{VIDA y OBRa de Trissino}

Messer Giangiorgio Trissino nació en Vicenza en 1478 en el seno de una familia patricia - de ahí el tratamiento de "Messer"-, y falleció en Roma en 1550. Recibió una esmerada educación humanística, estudiando además griego en Milán. Su amplia cultura le valió la atención del Papa León $\mathrm{X}$, quien en 1515 le envió a Germania como su Nuncio. También fue embajador del posterior Papa Clemente VII, que le mostró especial favor, e igualmente fue protegido por el Papa Pablo III. En 1530 sujetó, con otros notables, el manto imperial durante la coronación de Carlos V en Bolonia. Y en 1532, el Emperador Carlos V le otorgó el título de Conde Palatino.

En 1510 fue expulsado de su ciudad y de su República, Venecia, acusado de haber favorecido los planes anexionistas del Emperador Maximiliano I, pero su exilio en Germania duró pocos meses. Viajó por toda Italia (especialmente Milán, Florencia y Roma) y recibió honores. Ejerció el mecenazgo: donde quiera que establecía su hogar, éste se convertía en el centro de literatos y hombres cultos. Así, descubrió el talento del arquitecto Andrea Palladio ${ }^{3}$, estableciéndose entre ambos una larga y estrecha amistad, cuyo exponente fue la construcción, por Palladio, de "Villa Trissino" en Cricoli". Se casó dos veces: la primera a los dieciséis años, con su pariente Giovanna Trissino, y de ese feliz matrimonio nacieron cinco hijos. De ellos sólo sobrevivió uno, Giulio, a quien quiso tiernamente, pero él le proporcionaría grandes disgustos.

3 Realmente se llamaba Andrea DI PIETRO. El nombre renacentista de "PALLADIO" se lo sugirió el propio TRISSINO. PALLADIO es el nombre de un ángel en su L'Italia liberata da' Goti.

4 Cfr. BARBIERI, Franco: "Giangiorgio Trissino e Andrea Palladio", en Atti del Convegno di studi su Giangiorgio Trissino. A cura di Neri Pozza. Vicenza: Accademia Olimpica, 1980, pp. 191-211. 
Veinte años después de la muerte de su primera esposa, volvió a casarse con otra pariente, Bianca Trissino, con la cual tuvo dos hijos. Sus últimos años fueron amargos. Primero, por problemas con Giulio, Bianca se separó de su marido. Y después Giulio expropió la casa a su padre. Trissino va a Germania a pedir justicia al Emperador, vuelve a Roma, y muere allí. (B. Morsolin, 1894).

Fue hombre de letras de amplísima cultura. Su labor abarca varios campos, en la práctica literaria y en la teoría. Incluso dedicó su atención a la arquitectura, emprendiendo un opúsculo sobre este tema. Dentro de la creación literaria, cultivó la poesía (Rime, 1529), el teatro (tanto la tragedia: Sophonisba, 1513-14, publ. 1524, como la comedia: I Simillimi -basada en los Menaechmi de Plauto-, 1548), y el poema épico (L'Italia liberata da' Goti-que dedicó al emperador Carlos V-, 1547-48, tras veinte años de intenso trabajo) ${ }^{5}$. Como teórico de la literatura, es muy importante su Poética (en 1529 aparecieron los cuatro primeros libros o "divisiones", y en 1562 las divisiones quinta y sexta, póstumamente publicadas). La Poética es, pensamos, la primera aristotélica en Italia. Es, a la vez, el mejor tratado métrico italiano escrito hasta entonces, y seguiría siéndolo por mucho tiempo ${ }^{6}$. En este campo también, Trissino descubrió, tradujo y publicó el De Vulgari Eloquentia de Dante (en 1529 igualmente). Y en el ensayo biográfico, celebró a las claras mujeres en I ritratti (1524).

Igualmente son muy notables sus teorías lingüísticas. La cuestión del "volgare" o lengua vernácula comolengua literaria

5 WEINBERG, Bernard (A History of Literary Criticism in the Italian Renaissance, The University of Chicago Press, vol. I, 1961, pp. 368-370) señala traducimos nosotros- que "la primera utilización extensa de la Poética [de ARISTÓTELES] en Italia" se encuentra en TRISSINO, en la dedicatoria de la Sophonisba al Papa León X (1524).

6 Con posterioridad a la aparición de las cuatro primeras divisiones de la Poética de TRISSINO (1529), surgirían dos obras, usadas sobre todo como diccionarios de rimas: le Institutioni al comporre in ogni sorte di rima (1541), de Mario EQUICOLA, pero sobre todo el Rimario y el tratado Del modo di comporre in versi nella lingua italiana (1559), de Girolamo RUSCELLI. Este último autor, junto con RENGIFO y su Arte Poética Española (1592), influirían en la mejor y más amplia Métrica española y comparada de los siglos de Oro, cuya teorización supera ampliamente a sus modelos: la Rythmica, parte II del Primus Calamus, de Juan CARAMUEL (1665). 
moderna, y su fijación para toda Italia, era entonces candente objeto de debates. Polemista, Trissino expuso sus ideas -que parten de Dante y se despliegan originalmente- en varios lugares: en el diálogo Il Castellano (1529); en la Grammatichetta (1529); en la Epistola a Papa Clemente VII (1524); en Dubbii grammaticali (1529); en Epistola de le lettere nuovamente aggiunte ne la lingua italiana (1529), e incluso en la segunda división de la Poética (1529). Propuso una ampliación del alfabeto italiano a 33 letras (28 "significativas" y 5 "ociosas"), distinguiendo entre vocales medias abiertas y cerradas, y entre consonantes fricativas y africadas -diríamos hoy-. Aunque fonética y etimológicamente esta propuesta es correcta, no prosperó.

Enorme éxito y repercusión literaria tuvo la tragedia Sophonisba, con la que creó la tragedia clasicista. Y en el poema épico (llamado entonces "heroico") L'Italia liberata da' Goti-que narra la campaña de Belisario en Italia-, aplicó los principios aristotélicos frente a la libertad fantástica de los "romanzi" o épica caballeresca. Esta obra fue bastante atacada, a pesar de haber trabajado tanto en ella y ser su preferida. Sus Obras Completas fueron publicadas en Verona en 1729.

\section{EL "VERSO SCIOLTO", TIMBRE DE GLORIA DE TRISSINO}

Dentro del dinamismo literario y lingüístico de nuestro autor, además de su obra máxima, la Sophonisba, con la que renovaría totalmente el teatro italiano y el europeo, además de su descollante Poética, tenemos que referirnos a su otro timbre de gloria: su impulso definitivo al "verso sciolto". No es el primer poeta que lo usa, pero sí es el primero que lo utiliza para obras extensas (en la tragedia Sophonisba, y, sobre todo, en el poema épico -"heroico"-L'Italia liberata da' Goti, escrito íntegramente en verso suelto). Es, también, el primero que teoriza sobre él, justificándolo. El endecasílabo suelto, que tendría luego un uso amplísimo, tanto en la literatura italiana como en el resto de las europeas, y llegaría 
triunfante hasta nuestros días, se consolida y se extiende gracias a Giangiorgio Trissino ${ }^{7}$.

La tragedia Sophonisba es la primera obra que utiliza muy mayoritariamente el verso suelto ${ }^{8}$. Escrita en Roma en 151314, aparece publicada en 1524, dedicada al Papa León X. En previsión de ser atacado por esa novedad, se anticipa en la Epístola-dedicatoria a refutar los ataques: Afirma que no se le puede recriminar haberla escrito "en lengua italiana, y el no haber concordado las rimas según el uso común, sino haberlas dejado libres en muchos lugares" Nos da también la fundamentación psicológica para el verso suelto de la Sophonisba: dar naturalidad a los diálogos, y permitir que se expresen más libremente las pasiones.

$\mathrm{Y}$ en cuanto al no haber concordado las rimas totalmente, [es] (...) porque el lenguaje que mueve esta [tragedia] nace del dolor, y el dolor manda fuera impensadas palabras, por lo cual la rima, que indica pensamiento [reflexión], es verdaderamente contraria a la compasión.

El verso suelto acerca a un ideal estético de realismo pasional y de cotidianidad ${ }^{10}$. Por otra parte, dice bien Trissino al afirmar que ha dejado libres los versos "en muchos lugares": Sobre 2188 versos que contiene la obra, 1574 son endecasílabos sueltos. Con ellos el autor da forma, sobre todo, a los largos

$\overline{7}$ El verso suelto apareció fugazmente al final del Duecento, y fue usado esporádicamente en el Trecento y en el Quattrocento (Leon Battista ALBERTI); en el Cinquecento, TRISSINO es quien por primera vez alcanza a ver su importancia y significado, y además lo aplica a la totalidad o cuasi-totalidad de obras literarias. En la segunda parte del siglo, su uso se incrementará para traducciones y poemas didácticos. Y Torquato TASSO lo empleará en Il mondo creato.

Más tarde, en el Settecento, Gian Vincenzo GRAVINA y Scipione MAFFEI continúan con su teorización, polemizando contra los partidarios de la rima. Giuseppe PARINI lo emplea en Mattino y Mezzogiorno. En la segunda mitad del Settecento y primera del Ottocento, se afirma con fuerza (FOSCOLO, LEOPARDI, MONTI), y continúa en el Novecento (GOZZANO, UNGARETTI, MONTALE, etc.) El endecasílabo suelto representa, frente al verso rimado, la espontaneidad, la proximidad a la vida, la libertad. (PAZZAGLIA, Mario: Manuale di Metrica italiana. Firenze: Sansoni, 1990, pp. 116-117).

8 Cfr. PAGLIERANI, Franco (ed.): La Sofonisba di Giangiorgio Trissino, con note di Torquato Tasso. Bologna: Presso Gaetano Romagnoli, 1884.

9 Traducimos nosotros, en este pasaje y en los siguientes, para mayor facilidad del lector.

10 Como FOSCOLO y PASCOLI más tarde. 
parlamentos puestos en boca de mensajeros y criados, y también a buena parte de los diálogos. Pero el verso suelto no ocupa la totalidad de la tragedia, pues también encontramos silvas, y, excepcionalmente, una serie de heptasílabos sin rima. Ambas modalidades las utiliza sobre todo el Coro, cuyos cantos son más poéticos. Con esta variedad métrica, seguramente busca Trissino dar movimiento rítmico a la obra, pues coincide siempre el cambio de modalidad versal con cambios en la acción.

Además de que el "verso sciolto" permite expresar con naturalidad la pasión, existe otra importante razón cultural, en Trissino, para su uso en poemas extensos, tragedias o heroicos: es el que mejor traduce en italiano el verso épico de Homero y Virgilio. Nos lo explicita en la Poética:

El verso hexámetro, por otra parte, se adapta muy bien por ser más firme y más alto que los otros y por recibir mejor que cualquier otro verso las lenguas ${ }^{11}$ y las metáforas y las otras figuras, como se ve en Homero primero y después en Virgilio. Pero nosotros, al no recibir nuestra lengua esta clase de versos, hemos elegido el endecasílabo, el cual, por no concordar las últimas desinencias se llama "suelto"; y estas tales últimas desinencias, casi todos aquellos que han escrito en esta nuestra lengua italiana las han, hasta ahora, concordado de diversos modos, como en los libros precedentes hemos mostrado ${ }^{12}$.

Con este trasfondo homérico y virgiliano, Trissino escribe íntegramente en endecasílabos sueltos su poema heroico L'Italia liberata da' Goti, el primero en esta forma, y por tanto innovación absoluta. Cumple así Trissino su sueño de enlazar la literatura italiana con la griega y latina, y realizar en la épica lo que ya había hecho en la tragedia, recuperando así los principales géneros del Clasicismo griego. L'Italia liberata da' Goti, en 27 libros, fue su obra de más empeño. Según sus palabras, empleó en su escritura más de veinte años, y fue su obra preferida. Los nueve primeros libros de L'Italia liberata da' Goti aparecieron en 1547, y los dieciocho restantes

11 Son las $\gamma \lambda \omega \tau \tau \alpha$ de ARISTÓTELES (Poética, 1458 ), palabra traducida entonces habitualmente como "lenguas" (hoy, "palabras extranjeras o forasteras").

12 Sexta división, p. 25. 
en 1548. (Esto nos retrotrae, para calcular su comienzo, a 1527-28, antes de que fuera publicada la primera parte de la Poética). Se la dedica al Emperador Carlos V ("il piú virtuoso, et il piú degno Principe"), a quien Trissino considera el moderno liberador de Italia, el predestinado a realizar lo que era el sueño político de nuestro autor: plasmar el Sacro Imperio Romano, y unificar toda la Cristiandad frente al otomano. Su signo sería el águila, en paralelo con el único poder religioso, el Papa de Roma, bajo el signo de la cruz.

Además, para Trissino, sólo el endecasílabo suelto podía expresar el "continuum" de las acciones del poema heroico, mientras los tercetos encadenados dantescos o la octava real -el tipo que se usaba preferentemente y se usaría en Italia (y España) para este tipo de poemas-, imponían la pausa estrófica, la disrupción, cada ocho versos. Su propuesta del endecasílabo suelto para el poema heroico recibió algunas adhesiones y bastantes ataques ${ }^{13}$.

\section{LA POÉTICA}

La edición principal de la Poética de Aristóteles, la que el Renacimiento conocería y comentaría, apareció en 1508, juntamente con la Retórica del mismo Aristóteles y con el

13 Recibió las adhesiones de Girolamo MUZIO (Egida) y después de Gabriello CHIABRERA (Goteide). Tuvo también fortuna en la cultura francesa clasicista del siglo XVIII. Por otra parte, el verso suelto, gracias al ejemplo de TRISSINO, se difundió en el Cinquecento italiano por tragedias, comedias, además de otros géneros menores (églogas, elegía, sátira, sermón, epístola, poema didascálico, fábula mitológica, etc.). (Cfr. BAUSI, Francesco, y MARTELLI, Mario: La metrica italiana. Teoria e storia. Firenze: Casa Editrice Le Lettere, 1993, pp. 147-151).

En cambio, en el campo del poema heroico, los tercetos encadenados y la octava rima continúan empleándose. La octava rima se impone hacia la mitad del siglo y en adelante para los poemas épicos. Giraldi CINZIO, en el Discorso intorno al comporre dei romanzi ataca al verso suelto (1554); y Torquato TASSO, en Discorsi del poema eroico, libro VI, hace un elenco de los muchos poemas heroicos que se han escrito en octavas rimas. (Cfr. MARTELLI, Mario: "Le forme poetiche italiane dal Cinquecento ai nostri giorni", en Alberto A. Rosa (dir.): Letteratura italiana, vol. III, Le forme del testo. 1. Teoria e poesia. Torino: Giulio Einaudi editore, 1984, pp. 530-543). 
De elocutione de Demetrio Falereo ${ }^{14}$. En 1522, Abram de Balmes publica la traducción latina de la traducción de Averroes de la Poética de Aristóteles, y parafrasea esta Poética. En 1524, Trissino publica su Sophonisba, empapada de clasicismo y con referencias directas a la Poética de Aristóteles. Y en 1529 tenemos la Poética de Trissino.

Esta Poética consta de seis "divisiones" o libros. Presenta la peculiaridad de haber sido publicada de dos veces, en dos fechas muy separadas: 1529 (las cuatro primeras divisiones) y 1562, después de la muerte de Trissino (1550) (la quinta y la sexta divisiones). Si consideramos la fecha de aparición de su primera parte, podemos decir que es la primera poética aristotélica en lengua italiana (1529): la primera que toma a Aristóteles como punto de partida y como guía para su propia reflexión ${ }^{15}$.

La redacción última de la quinta y sexta divisiones, sin embargo, debían estar completadas alrededor de $1949^{16}$. Esto, y la neta distinción temática entre ambas, ha dado pie a que se considere por algunos autores -erróneamente, creemos- que sólo es de impronta aristotélica el segundo libro, mientras el primero sería un "Ars Versificandi" emparentado con la Retórica, fuera de la órbita aristotélica.

Personalmente, consideramos que la Poética de Trissino es una, y no dos; y que hay una cohesión, un diseño perfecto de sus contenidos, escalonados; no una simple superposición de conocimientos. Trissino conocía ya la Poética de Aristóteles en 1514-1515, fecha de composición de su Sophonisba, puesto que ajusta su escritura las normas aristotélicas. Además, habla explícitamente de Aristóteles en la Epístola-Dedicatoria de esta obra al Papa León X (1524).

${ }_{14}$ Con anterioridad a esta fecha tenemos, en Italia: en 1481, primera edición de la traducción latina de la Paráfrasis a la Poética aristotélica de AVERROES, hecha por HERMANNUS ALEMANUS. Y en 1498, la traducción latina de la Poética, hecha por Giorgio VALLA. Como señala WEINBERG, Bernard ( $A$ History of Literary Criticism in the Italian Renaissance, cit.), ignoramos cuál de los ejemplares aristotélicos manejó TRISSINO.

15 En la Poética, justo en su comienzo ("primera división”), TRISSINO señala sus objetivos parafraseando a ARISTÓTELES, y vuelve a mencionar al Estagirita más adelante, a propósito de los extranjerismos.

16 WEINBERG, Bernard: A History of Literary Criticism in the Italian Renaissance, cit. (vol. I, pp. 368-70, y vol. II, pp.750-55 y 1070-71), II, p. 750. 
Y sabiendo además que la Tragedia, según Aristóteles, es antepuesta a todos los otros poemas, por imitar con suave lenguaje una virtuosa y perfecta acción, que tenga grandeza (...) así la Tragedia, imitando, hace a los caracteres mejores, y la Comedia peores. Y por eso la Comedia mueve a risa, porque participa de la fealdad, ya que lo ridículo es defectuoso y feo. Pero la Tragedia mueve a compasión y temor; con ellos, y con otras enseñanzas, aporta deleite a los oyentes, y utilidad al vivir humano. (...)[La tragedia tiene] seis partes necesarias, a saber, la Fábula, los Caracteres, las Palabras, el Lenguaje, la Representación, y el Canto.

En la Poética, en la primera división, p. $2 v$, justo en el segundo párrafo, ya anuncia sus intenciones -y muestra la órbita aristotélica en la que está, y que desarrollará ampliamente en las dos últimas divisiones. Obsérvese el enlace que Trissino establece entre la teoría aristotélica, generalista, de primer nivel, y la teoría del lenguaje poético, de segundo nivel, más específica, que va a exponer a continuación:

Digo, pues, que la Poesía (como antes dijo Aristóteles) es una imitación de las acciones del hombre ${ }^{17}$; y haciéndose esta tal imitación con palabras, rima y armonía, al igual que la imitación del pintor se hace con dibujo y con colores, será bueno, antes de venir a esa imitación $^{18}$, tratar de aquello con lo que la imitación se hace, es decir, de las palabras y de los versos, dejando aparte la armonía o el canto, ya que aquéllos pueden hacer la imitación sin éstos, y aquellos dos el poeta los examina, dejando al cantor examinar el canto. Comenzaré, pues, con la elección de las palabras, y después hablaré de los versos, en los cuales me extenderé un poco, por no haber sido en nuestros tiempos tan bien entendidos como lo fueron en los tiempos de Dante, y de Petrarca, y de otros buenos autores. De las razones y el uso de palabras y versos no me apartaré, y para una demostración más clara, quiero servirme de ellos donde quiera que se necesiten ejemplos.

Efectivamente. La primera división trata de la elección de la lengua; la segunda, del ritmo de los versos; la tercera, de las rimas; y la cuarta, de los poemas vigentes en su época. Al finalizar la cuarta, anuncia que faltan dos divisiones por publicar, que tratarán de la tragedia, la comedia, el poema heroico y elementos comunes a todos los poemas (serán las

${ }_{17}$ Poética, 1448a2.

18 A la teoría del teatro y del poema épico. 
figuras de estilo). Vemos, pues, el perfecto dibujo de la teoría poética en la mente y en el proyecto de Trissino. Y vemos que la Poética de Aristóteles es el marco donde Trissino inserta su propia teoría del lenguaje poético.

Naturalmente, dada la gran cultura de nuestro autor, además de Aristóteles, otras fuentes cooperan en el levantamiento del edificio: Horacio, Dionisio de Halicarnaso y Hermógenes son las principales ${ }^{19}$. Para Trissino, hombre del Renacimiento, sería absurdo contraponerlas entre sí; por eso lo que hace es sumarlas, recogiendo de cada autor lo que le parece que explica la realidad de la Poesía. De Aristóteles toma el encuadre general de sus teorías, $y$, más señaladamente, en las divisiones V y VI toma lo que Aristóteles desarrolló: la teoría de la tragedia y la del poema épico. En cambio, para lo que Aristóteles no trató, la teoría de la lírica, Trissino recurre por un lado a su propia experiencia, y por otro a sus lecturas. Va ensamblando sus observaciones personales y sus fuentes con habilidad, buscando siempre la verdad. Así esta Poética ordenadísima y muy didáctica da un sólido cuerpo a una teoría literaria total.

\section{LA TEORÍA MÉTRICA}

5.1. Una gran coherencia preside la Poética. Ya en la primera división y en su comienzo (pp. 2 y $2 v$ ), Trissino nos enuncia su propósito: Tratar de la Poesía en lengua italiana -entendiendo la Poesía en sentido amplio, como Aristóteles y los griegos: la creación literaria en todas sus formas (géneros), y expresada en verso. Puesto que la Poesía en lengua griega y latina ya ha sido tratada ampliamente,

me ha parecido bien dar yo ésta [la Poética] a nuestra lengua italiana. En la cual, si bien se ha escrito por muchos poéticamente, y con arte, nadie hasta ahora ha tratado de su Arte, excepto Dante y Antonio de Tempo, los cuales escribieron en latín casi al mismo tiempo ${ }^{20}$. Pero

19 Cfr. VEGA, María José: “La Poética hermogénica renacentista: Giovan Giorgio Trissino". Rev. Castilla (Universidad de Valladolid), 1991, 16, pp. 169-188.

20 Se refiere al De Vulgari Eloquentia, que el propio TRISSINO tradujo y publicó 
yo escribiré en nuestra lengua, [y] espero tratar más copiosamente y más distintamente que ninguno de ellos $^{21}$; por eso guardaré otro orden y trataré de todas las partes de la Poesía, las cuales en aquella edad no eran conocidas y, seguramente por esta razón, fueron abandonadas por ellos.

A continuación - como ya hemos visto-menciona Trissino explícitamente a Aristóteles, definiendo la Poesía como "imitación de las acciones del hombre" ${ }^{22}$, imitación que se hace con palabras, ritmo y armonía ${ }^{23}$. Por ello, decide que va a empezar "con la elección de las palabras, y después hablaré de los versos". Efectivamente, a cuestiones lingüísticas dedica Trissino la primera división (elección de la lengua, elección general de las palabras, elección particular de las palabras, formas de decir - claridad, grandeza, belleza, velocidad, caracterización, verdad, artificio-, pasiones de las palabras -superabundancia, carencia, mutación y transposición-. Acompaña cada objeto de estudio con el correspondiente ejemplo (o ejemplos) de grandes poetas.

5.2. La segunda división aborda ya plenamente el estudio del ritmo. Y hemos de señalar la gran originalidad de Trissino, dado que nadie antes de él había abordado para la lengua italiana estas cuestiones, y además lo hace con gran profundidad y minucia. Define el ritmo "de la voz articulada" como "una resonancia que resulta de cierta cantidad y cualidad de sílabas, puestas juntas con orden y terminadas

en 1529, como ya hemos dicho, y al Summa Artis Rithimici Vulgaris Dictaminis de Antonio de TEMPO (o da Tempo) (1332). En realidad, el de TEMPO es unas tres décadas posterior al de DANTE, pero no lo conoció, y por tanto TEMPO innovó totalmente.

21 Efectivamente: DANTE en su importante, breve e incompleto tratado, aborda una gran diversidad de temas. Anuncia que va a tratar de la canción, el soneto y la balada, pero al poco de comenzar con la canción -haciendo muy importantes observaciones- se interrumpe el libro. Lástima. Por su parte, la notable obra de Antonio da TEMPO censa 7 tipos poemáticos (con sus numerosas variantes). Presta atención a la rima y al número de sílabas, y se interesa por todo un conjunto de juegos de palabras que aparecen en la poesía. Pero no trata en absoluto otras cuestiones métricas como el acento, ni tampoco otros géneros poéticos (tragedia, poema épico, etc.).

22 Poética 1448a.

23 Ibid., 1447a. 
con orden." Por ello comienza tratando "de las letras" -tema éste que había abordado ampliamente en otras obras, y que es muy original, pues aumenta el alfabeto italiano a treinta y tres letras (veintiocho "significativas" y cinco "ociosas"). De las veintiocho significativas, siete son vocales y veintiuna consonantes. Esto le permite distinguir -diríamos hoy-entre vocales medias $(\mathrm{e}, \mathrm{o})$ abiertas y cerradas, y entre consonantes fricativas y africadas.

Tras el muy sintético estudio de las letras, aborda las sílabas, los acentos, y finalmente recala en los "pies". Con las letras se hacen las sílabas, y con las sílabas los pies. "Y estos pies son los que gobiernan los versos, los cuales con ellos casi caminan." Considera que, a diferencia de las lenguas clásicas, los pies trisílabos no son útiles en los poemas italianos, por lo cual se centra en los bisílabos: yambo, troqueo, espondeo y pirriquio. (El espondeo, de dos sílabas "agudas" -tónicas- sólo podrá encontrarse en dos palabras consecutivas, y el pirriquio, de dos sílabas "graves" -átonas-, sólo en interior de palabra polisílaba). En realidad, son los yambos y los troqueos los que centrarán su sistema.

De la unión de los pies resultan las "medidas" o metros (como en la métrica clásica). Atendiendo a la terminación de los versos, los trocaicos tienen dos medidas, y cada medida dos pies: son "dímetros". Y los yámbicos pueden tener dos o tres medidas. En este caso serán "trímetros". Puede haber algún "monómetro", pero raramente. También encuentra dieciséis medidas "cuadrisílabas", que denomina con la terminología clásica (ditroqueo, peones, epítritos, jónicos, etc.), y que aparecen algunas veces en los versos italianos. Para mayor claridad en la teoría de las medidas en Trissino, digamos que el verso italiano más frecuente es el "trímetro" (endecasílabo); y el segundo más frecuente el "dímetro" (heptasílabo).

El resto de la división es un detallado estudio sobre "cuáles deben ser las primeras medidas" de los versos, cuáles las segundas medidas y las terceras. También aborda los versos trocaicos, que "aunque no se encuentren ni en Petrarca ni en Dante", sí aparecen a menudo entre los primitivos sicilianos y algunos stilnovistas. Habla de la "remoción, colisión y 
pronunciación conjunta", tres fenómenos que disminuyen el verso en una sílaba, aunque en realidad sean distintos. Y termina examinando las "cesuras" del verso, que son -recordando a las clásicas, aunque siempre entre palabras completasla quinta y séptima cesura. De todo ello aporta suficientes versos ejemplificadores, siempre endecasílabos.

5.3. La tercera y la cuarta divisiones son las más largas, originales e inolvidables. En la tercera estudia el fenómeno de la rima (que él casi siempre llama "las desinencias", puesto que la palabra "rima" era polisémica en esos siglos $\left.{ }^{24}\right)$. Comienza distinguiendo entre lo que hoy llamamos versos agudos, llanos y esdrújulos en cuanto a la rima, y que él denomina "versos menguados, simples y llenos". (Nótese el esfuerzo de Trissino para crear un lenguaje específicamente métrico, para configurar toda una terminología que fije los conceptos de este nuevo campo de estudio. Éste es, precisamente, uno de los grandes aportes de su Poética).

Y a continuación, dentro de lo que hoy llamamos estrofa y Trissino llama "combinaciones" o "cuadrillas" de versos que obedecen a un patrón, va a explorar los "modos" o esquemas de rimas para la agrupación de los versos según las desinencias. La agrupación más sencilla es la "pareja" de versos (tanto en los versos menguados como en los simples y llenos). La pareja puede tener terminación "concorde" (puede rimar) o "discorde" (no rimar). De ambos aporta ejemplos de Petrarca con un esquema de rimas en su margen derecho, y añade al final el resumen de los dos (aa /ab) y una tabla donde el esquema se visualiza. (Obsérvese que nadie antes de Trissino -que sepamos nosotros- había hecho esto, cuyo carácter didáctico y mnemotécnico le ha permitido permanecer hasta nuestros días en nuestros manuales).

Después vienen los “tercetos", a los que aplica el mismo método: ejemplos (de Petrarca o de otros) con el correspondiente

${ }^{24}$ Recordemos, p. ej., de PETRARCA, las Rime in vita di Madonna Laura; de LOPE DE VEGA, las Rimas humanas; etc. Cfr. DIETRICK, Déborah: "De consonancia a rima", en Antonio Álvarez Tejedor y AA. VV: Lengua viva. Estudios ofrecidos a César Hernández Alonso. Valladolid: Universidad de Valladolid, 2008, pp. 1055-1073. 
esquema en el margen derecho, y finalizando con la tabla de los "modos" de rimas, que ahora son ya cinco: abc - aba $a b b-a a b-y$ aaa. Y aún mayor complejidad de modos poseen los "cuaternarios", pues suman quince: abba - abbc - abbb $a b a b-a b a c-a b a a-a b c d-a b c a-a b c b-a b c c-a a b c-a a b a$ - aabb - aaab - y aaaa. Ejemplos de Petrarca, Dante, Cino da Pistoia, Giacomo da Lentini, Guittone d'Arezzo, Bonagiunta da Luca, el rey Enzo de Sicilia, Messer Honesto y otros ilustran estos modos.

La combinatoria de los "quinarios" y "senarios" aumenta extraordinariamente (p. ej., cincuenta y dos modos para los quinarios). No obstante, "se usan pocos modos de quinarios, y esos pocos rarísimas veces". Por lo cual reproduce los modos de los quinarios encontrados, que son cinco: abbcd - abced - aabbc - ababc - y ababb. Y el de los "senarios", dos: aabbcd - y abbccd. "Más allá de los senarios no se encuentra ninguna combinación, ni de septenarios, ni de octonarios o similares". Ante la imaginable sorpresa del lector, en seguida matiza: "aunque se encuentran sirimas de canciones con mayor número, y así he visto algunas de hasta dieciocho versos." La canción de estancias parece plantear problemas en este punto a Trissino, pues continúa matizando, zigzagueando, y tirando finalmente por la calle del medio:

Se pueden encontrar estancias continuas con un número mayor; pero aunque una responde a la otra, ésta no es, sin embargo, una verdadera combinación, como veremos al tratar de ellas. No obstante, digo que no se encuentra combinación con más de doce versos, es decir, dos senarios. Y los senarios, por ser de uso rarísimo, dejaremos que los formen quienes quieran usarlos ${ }^{25}$.

A continuación habla de las estrofas, que él denomina "combinaciones". Define la combinación como "un poner juntos parejas, tercetos, cuaternarios y demás, que sean del

\footnotetext{
${ }_{25}$ Cursivas nuestras. Pensamos que la dubitación trissiniana en torno a la canción de estancias se debe a que, efectivamente, la canción de estancias no es un poema estrófico realmente, sino una forma fija, aunque puede -y suele- repetir el esquema de la primera estancia. (Cfr. PARAÍSO, Isabel: La Métrica española en su contexto románico. Madrid: Arco/Libros, 2000, pp. 325-329.)
} 
mismo modo." Esto implica que, p. ej., un terceto del primer modo sólo puede juntarse con otro del mismo modo, no de un modo distinto. Además, los versos "deben ser de la misma cualidad", o sea, si un terceto tiene un dímetro y dos trímetros, así el segundo y los demás. (Nótese cómo en este punto engarza Trissino la teoría de las medidas, examinada en la segunda división. Recordemos que el "dímetro" es nuestro heptasílabo, y el "trímetro" el endecasílabo). Así pues, para que existan estrofas se precisa el mismo esquema de rimas y la misma disposición de los metros, si hay heterometría. Además, si hubiera "desinencias en las cesuras" (lo que luego se llamará "rima al mezzo"), la combinación (estrofa) exige que su esquema se mantenga a todo lo largo del poema. Todo ello se encuentra suficientemente ejemplificado.

Luego examina las "combinaciones completamente discordes en las rimas", las "concordes en todo" y las "discordes en parte"; es decir, las estrofas que cambian de rima (p. ej. abba - cddc), las que no cambian (p. ej. abba - abba), y las que cambian parcialmente (p. ej. abc - adc). En las combinaciones concordes en todo, distingue entre una concordancia "recta" $(\mathrm{ab}-\mathrm{ab})$ y otra "oblicua" $(\mathrm{ab}-\mathrm{ba})$.

Al mismo tiempo, aplica esta disposición de rimas "recta" y "oblicua" a los "modos" previamente vistos (parejas, tercetos, etc.), obteniendo toda una teoría combinatoria de las rimas. Como muestra de ella, que es bastante extensa, resumiremos aquí solamente su aplicación a las estrofas "concordes", y, dentro de ellas, a las parejas y los tercetos. Para los cuaternarios, el propio Trissino selecciona, porque "ponerlos todos (además de que no los he encontrado todos en uso) sería una cosa demasiado larga". Y de los quinarios y senarios también selecciona, "por no estar (como he dicho) todos en uso; y las que existen, son las rectas".

El primer modo de pareados o "parejas" $(a b)^{26}$ tiene dos combinaciones concordes: una recta $(a b-a b)$ y otra oblicua

26 Aunque habitualmente hoy representamos con letra mayúscula los versos de arte mayor, y en los ejemplos solemos tener endecasílabos, seguiremos aquí y en adelante la anotación de TRISSINO, que utiliza solamente la letra minúscula para las rimas. 
$(\mathrm{ab}-\mathrm{ba})$. El segundo modo $(a a)$ no tiene combinación concorde por tener la misma desinencia. El primer modo de tercetos $(a b c)$ tiene seis combinaciones concordes: una recta $(a b c-a b c)$ y cinco oblicuas: la primera $(a b c-b a c)$; la segunda $(a b c-c b a)$; la tercera $(a b c-a c b)$; la cuarta $(a b c-b c a)$; y la quinta $(a b c-c a b)$. El segundo modo de tercetos $(a b a)$ tiene dos combinaciones concordes en todo: una recta (aba - aba) y una oblicua (aba - bab). El tercer modo $(a b b)$ tiene igualmente dos combinaciones: una recta $(a b b-a b b)$ y otra oblicua ( $a b b$ - baa). El cuarto modo ( $a a b)$, lo mismo: una recta $(a a b-a a b)$ y otra oblicua $(a a b-b b a)$. Y el quinto y último modo $(a a a)$ no tiene combinación concorde. Naturalmente, ejemplifica cada uno de estos casos.

En las combinaciones "discordes en parte" también encuentra, a partir de los tercetos, diversos modos, y dentro de ellos combinaciones rectas y oblicuas. Ahorraremos su exposición, bastante amplia.

Termina la tercera división tratando de las "mezclas", que son lo opuesto a las "combinaciones": Si éstas consisten en reunir juntos dos tercetos, p. ej., del mismo modo, las mezclas consisten en reunir juntos dos tercetos de modos distintos (p. ej., abc-cbb).

5.4. La cuarta división es, sencillamente, prodigiosa. Aquí Trissino presenta con el mayor cuidado, sensibilidad y conocimiento, el panorama de todos los tipos poemáticos usados en ese momento; por eso es la división más amplia. Se apoya en Dante y en Tempo, pero supera con mucho, en profundidad de análisis, sus modelos.

Frente a la única forma estudiada -de manera magnífica e incompleta- por Dante, la canción; y frente a las siete estudiadas por Tempo ${ }^{27}$, Trissino aborda cinco: soneto, balada, canción, madrigal y "serventese" (que no se corresponde con

27 Recordemos que DANTE se había propuesto tratar de los "modos" más dignos de la poesía italiana: canción, balada y soneto, dejando para el final algunos modos "ilegítimos e irregulares"; pero, de hecho, dado que el De Vulgari Eloquentia está incompleto, sólo estudió parcialmente la canción. Por su parte, Antonio da TEMPO estudia siete "genera": "sonetus", "ballata", "cantio extensa", "rotundellus", "mandrialis", "serventesius" y "motus confectus". 
nuestro serventesio). Descarta, con razón, las otras dos que Tempo también abordaba: el "rotundellus" (que no equivale a nuestra redondilla, sino a nuestro zéjel; lo descarta porque no lo encuentra "en uso entre los buenos autores"; y con razón, pues históricamente es muy anterior y no podía estar "en uso"). Además, descarta el "motus confectus" con muchísima más razón, pues es un "unicum", un tipo de poema del que sólo se conserva el ejemplo que da el propio Tempo, y que con toda probabilidad es obra suya. Algún estudioso italiano ha llegado a pensar si sería una broma del autor ${ }^{28}$.

5.4.1. El estudio del SONETo viene en primer lugar, como en Tempo ${ }^{29}$. Da su etimología ('canto pequeño') y su estructura: dos combinaciones, una de cuaternarios y otra de tercetos ${ }^{30}$. Se aparta Trissino de Dante, que las denominó "pies" y "versos", para llamarlas "bases" y "vueltas". Tiene presente el estudio morfológico que Tempo hace de los sonetos, especialmente del simple, pero Trissino va mucho más lejos, estudiando por una parte las bases y por otra las vueltas. Y, lo que es novedad absoluta en Trissino, dedica un epígrafe al "unir las vueltas a las bases", es decir, si entre las bases y las vueltas hay o no continuidad de rimas.

28 BAUSI, Francesco, y MARTELLI, Mario (La metrica italiana, cit., p. 114) observan el interés de TEMPO por las formas métricas "irregulares" e incluso bizarras. Por nuestra parte, veamos resumidamente y con terminología adaptada los principales rasgos del "motus confectus". Se llama "confectus" porque está compuesto con sentencias muy notables y bellas; y "motus" porque mueve al hombre de limpio intelecto. Algunos falsamente los llaman "frotolas", obra de rústicos, sin sentencias. Consta de endecasílabos y heptasílabos, o bien de dodecasílabos, o bien de otras medidas. Forman series de versos monorrimos (de tres en tres, o de más o de menos). De todos modos, no tiene reglas fijas para los versos ni las sílabas, pero debe mantener la consonancia consecutiva. (TEMPO, Antonio da: Summa Artis Rithimici Vulgaris Dictaminis (1332; nueva publ. Venezia, 1509). Edizione critica a cura di Richard Andrews. Bologna: Commisione per i Testi di Lingua, 1977, cap. LXI, pp. 81-85).

29 Ibid., pp. XXXVIIv-XLI.

30 No se le escapa a la cultura de TRISSINO alguna otra estructura. Así la que hoy se conoce como "soneto shakespeariano" $(3+3+3+2$ versos): "No se me oculta que en algunos antiquísimos autores, antes de la edad de DANTE, se encuentran algunos sonetos que tienen tres bases de tres cuaternarios; no obstante, pienso que estas tales bases no deben imitarse, porque PETRARCA y DANTE y los otros buenos autores de aquella edad nunca los usaron". 
Las bases suelen ser de combinación recta y concorde, y pertenecer al primer modo de cuaternarios $(a b b a-a b b a)$, o bien de combinación recta y concorde, del cuarto modo de cuaternarios $(a b a b-a b a b)$. Encuentra otras tres, "de uso rarísimo": abab-baba; abab - baab; y abbb - baaa. De todos estos tipos da ejemplos, como de costumbre. En cuanto a las vueltas, Tempo había encontrado cuatro; Trissino encuentra muchas más, pero va a centrarse en seis, las más usuales, ejemplificándolas: Las tres primeras, $a b c-a b c$ y $a b c-b a c$ (recta y oblicua del primer modo); y $a b a-b a b$ (oblicua del segundo modo), son "de uso frecuentísimo". De uso no frecuente son otras tres: $a b a-a b a$ (recta del segundo modo); $a b b-a b b$ y $a b b-b a a$ (recta y oblicua, respectivamente, del segundo modo). En cuanto a las otras combinaciones, "se usan rarísimamente", por lo que las deja de lado, además de que ya han sido en parte tratadas en la tercera división.

Aborda también Trissino en epígrafe independiente el tema de la posible heterometría en los sonetos ("Del poner dímetros en los sonetos"). Tempo había dedicado un amplísimo estudio al soneto (caps. IV-XXXIV), en el que distinguía dieciséis "especies": "simplex, duplex, dimidiatus, caudatus, continuus, incatenatus, duodenarius, repetitus, retrogradus, semiliteratus, bilinguis, mutus, septenarius, communis et retornellatus", cada una de las cuales admitía subdivisiones y por tanto más tipos. Rengifo recogería en gran medida estas especies; pero a Trissino en cambio no le merecen mucha atención: Les dedica un epígrafe ("De algunas otras clases de sonetos"), y considera - con razón- que son formas de los poetas antiguos. En honor a algunos de éstos, que él estima (Guittone d'Arezzo, Guido Cavalcanti, Cino da Pistoia, Boccaccio, Burchiello), describe brevemente ciertos tipos (sonetos dobles, caudados, y con verso de cierre o "tornello"), mientras por los demás sobrevuela.

5.4.2. La BALADA ocupa un amplio espacio en la cuarta división (pp. XLIV-L), más del doble que el soneto. Después de explicarnos su etimología ("canciones que antiguamente se bailaban"), comienza Trissino comparando la 
estructura de la balada con la del soneto, y observando sus diferencias: poema más extenso, tiene una estructura más compleja: Las combinaciones pueden ser de cuaternarios, parejas, tercetos, quinarios o senarios, concordes o en parte discordes. El orden también es diferente: En primer lugar viene la mitad de la primera combinación ("represa"); en segundo lugar, la segunda combinación íntegra ("mudanzas"); $\mathrm{y}$ en tercer lugar, la otra mitad de la primera combinación ("vuelta").

A diferencia de Tempo, el gran teorizador de la balada, que estudia en primer lugar las baladas "grandes", luego las "medias", después las "menores" y por último las "mínimas", Trissino comienza por examinar las baladas "pequeñas": las que tienen represa de parejas (con rima $a b$ o bien $a a$ ). Después vienen las "medianas": las que tienen represa de tercetos (generalmente $a b a$, o bien $a b b$ ). Luego vienen las "grandes": las que tienen represa de cuaternarios (comúnmente $a b b a, 0$ $a b b c)$. Y por último las "mínimas": las que tienen como represa solo un verso $(a)$. A estas últimas les dedica poco espacio, por considerar que equivalen a un cuaternario del primer modo, se encuentran raramente, y casi siempre aparecen "replicadas", repetidas en su estructura. Precisamente este punto le lleva a tratar, en epígrafes propios, diversos casos: el de las baladas "replicadas" (con independencia de que sean mínimas, medias o grandes); el de las baladas que tienen dos vueltas; y el de algunas baladas que tienen tres mudanzas. Cada uno de los tipos de balada y de los casos especiales es examinado con minucia, atendiendo no sólo a sus represas sino también a sus mudanzas y vueltas. Recoge numerosas variantes, y las visualiza con abundantes ejemplos (de Guido Novello, Girardo da Castello, Petrarca, Cino, etc.).

5.4.3. En tercer lugar aborda la CANCión (la que Tempo llamó "cantio extensa", y Dante simplemente "canzone"). Trissino considera acertadamente que su "arte" es la estancia. Ésta permite establecer una primera división entre un tipo de canción de "estancia continua" (llamada también por el vulgo "sextina", dice el autor-denominación que ha prosperado-), 
y otro tipo, muchísimo más usado, de "estancia dividida". Hay que señalar que Tempo no hablaba en absoluto de la sextina -tal vez por desconocimiento-, y Dante, a pesar de conocer muy bien la obra de Arnaut Daniel, el creador, y a pesar de haber escrito él mismo algunas de las más inolvidables sextinas de la literatura italiana ${ }^{31}$, pasa por ella un tanto deprisa, cerca del final (II, XIII), al tratar de las rimas; probablemente por el carácter incompleto del capítulo y del libro. Por eso hay que agradecer a Trissino que sea el primer teorizador que estudia la sextina con la debida extensión, y que detalle sus reglas.

Tras estudiar la estructura-tipo y algunas variantes de la sextina usadas por Boccaccio y Petrarca, se centra en la "estancia dividida" -la que hoy llamamos "canción de estancias". Señala sus dos partes, tomando como referencia la " $d i$ visión" (el verso que después será conocido como "chiave" o llave; Dante lo llamó "diesis", y afirma que el vulgo lo llama "volta").

La parte que va desde la división hacia arriba, puede ser simple o repetida. Si es simple, se llamará "frente", y constará de un cuaternario, quinario o senario. Si es repetida, tendrá combinación de parejas, tercetos, cuaternarios, quinarios o senarios. Dante llamó a estas dos partes de la combinación "pedes", pies -nombre que ha perdurado-, pero Trissino prefiere llamarlas "bases", porque son apoyo y fundamento de toda la estancia. La segunda parte, desde la división hacia abajo, puede ser también simple o repetida. Si es simple, se llama "sirima". Si es repetida, o sea, tiene combinación, Trissino llama a sus dos partes "vueltas" (aunque Dante las llamó "versus"), para rehuir el equívoco.

Con la sistematicidad y orden que le caracterizan, Trissino aborda el estudio de la primera parte, tanto la simple como la dividida: primero la "frente", y después las "bases". Dentro de éstas, examina las bases de parejas, de tercetos, de cuaternarios, de quinarios y senarios, con abundantes ejemplos. Luego pasa a exponer las vueltas, y se centra en la sirima o

31 Como "Al poco giorno, e al gran cerchio d'ombra", que menciona el propio DANTE en el De Vulgari Eloquentia. 
parte simple -mucho más habitual que la otra posibilidad-, prestando atención especial a la "concatenación" o encaje, semántico y de rimas, entre los núcleos de la sirima, de tal modo que se engarcen pero no se parezcan a las combinaciones. (Obsérvese la sutileza de los análisis de Trissino). Luego dedica epígrafe especial al ensamblaje de vueltas y sirimas con las frentes y bases precedentes.

A continuación, pasa a examinar el conjunto del poema (canción de estancias): cómo las estancias deben ponerse juntas, y cómo debe cerrarse el poema. Y en este momento va a ser la rima (el "concordar de las estancias") el centro del estudio. Retoma la división entre "estancia continua" (sextina) y "estancia dividida". Comienza por la primera, describe su hermosa combinatoria, y la ejemplifica con esa extraordinaria sextina de Dante "Al poco giorno e al gran cerchio d'ombra". Las letras en el margen derecho, como siempre, explican gráficamente el complejo tejido de las palabras-rima. También explica las peculiaridades de la rima en las estancias divididas.

Al número de las estancias dedica un breve apartado puesto que el número es potestativo-, pero señala que no suelen ser ni menos de tres ni más de siete; como rarezas, anota una de Petrarca (10 estancias) y otra de Dante (15). Y por último dedica un largo apartado a los "cierres" de las canciones. Comienza con el cierre de la sextina, cuya breve estancia final de tres versos recoge las seis palabras-rima (como decimos nosotros, o, como dice Trissino, "las palabras últimas de las estancias") "dos por verso, una al final y otra en las cesuras". La canción de estancia dividida puede cerrarse con otra estancia de estructura igual a las demás; o bien la estancia final es más breve, y repite la estructura de la sirima. Los tres ejemplos que aporta son obra de su admirado Cino da Pistoia.

5.4.4. La cuarta forma que Trissino trata es el MADRIGAL. Para su etimología, recoge las disquisiciones de Tempo y las resume: "son llamados así porque en ellos se solían cantar cosas de amor, pero rupestres y pastoriles, casi apropiadas para los rebaños". Y en seguida describe su estructura: una combinación de dos o tres tercetos disímiles. Pueden ser 
concordes en todo, o discordes en todo, o en parte concordes y en parte discordes. Están a menudo rematados por dos "tornelli" o versos de cierre (un pareado). Sobre la posible existencia de dímetros (heptasílabos) -algo que Tempo recoge y elabora ampliamente-, entre los normales trímetros, Trissino señala que seguramente se pueden poner, pero "ya que los buenos autores no los han puesto, no me atrevo a decir que estén bien." (Como podemos constatar, el criterio de los "buenos autores" o modelos es esencial para Trissino). Los ejemplos pertenecen a Petrarca, Franco Sacchetti y Boccaccio.

5.4.5. La quinta y última forma abordada por nuestro autor es el "SERVENTESE". El nombre italiano nos hace pensar en el "sirventés" provenzal, e incluso en nuestro "serventesio"32; pero la estructura métrica es completamente diversa. Tempo nos suministra amplia información sobre el nombre y temática del "serventesius" o "sermontesius"; subraya la variabilidad de su composición, dejada al libre albedrío del poeta, y su pertenencia al estilo humilde ${ }^{33}$. Trissino, que tiene en mente la Divina Comedia de Dante y los Triunfos

32 Recordemos que el sirventés es una forma provenzal de canción que suele retomar el esquema métrico y la melodía de una canción preexistente (BELTRAMI, Pietro G.: La metrica italiana. Bologna: Il Mulino, $2^{\text {a }}$ ed. 1994, p. 364). El "serventese" italiano, según la tratadística antigua, comprende varias formas métricas cuyo contenido es didáctico, moralizante, cronístico o de ocasión. Coincide con el sirventés provenzal en ser poesía de ocasión o político-moral, pero no en la forma métrica.

33 "Et certe serventesius ideo dici potest quia servit quasi omnibus modis rithimandi supradictis; nam participat cum omnibus, ut ex eorum partibus versibus et sillabis infra patebit. Posset non improbabiliter dici quod ideo vocatur serventesius quia servit omnibus hominibus, et non habentibus subtiliorem intellectum, scilicet mechanicis et rusticis. [...] dummodo serventesius non sit historiographus seu figuratus ex historiis vel gestis antiquis subtiliter, quemadmodum fuit modus magistri Dantis Algerii. [...]

Quidam vero appellant hunc modum rithimandi sermontesium; et melius iudicio meo, quasi a sermone, quia sermo quidam vulgaris et non subtilis aliquorum verborum moralium in ipso continetur, vel gestorum antiquorum vel praesentium cum rithimis et consonantiis. [...]

Potest itaque serventesius compilari et fieri undenarius et septenarius et polysillabus brevis in rithimis, et multis aliis modis secundum beneplacitum rithimantium". 
de Petrarca para ilustrar esta categoría, perfila mucho más la estructura del "serventese": habitualmente son tercetos del segundo modo, en combinación oblicua y en parte discorde. $\mathrm{O}$, dicho con palabras de hoy, son, habitualmente, "tercetos encadenados" ( $a b a-b c b-\ldots y z y z)$; excepcionalmente, son otras formas encadenadas de tercetos y cuartetos.

Para el vicentino, dos notas lo caracterizan: es muy fácil, y es muy largo ("lo mismo que sobrepasa a todos los demás poemas de fácil composición, así también es el más largo entre ellos.") Tanto, que a menudo se divide en partes, que han sido llamadas "cantos" o "capítulos" 34 . Como de costumbre, incluye ejemplos: uno del "serventese" modélico (Dante) y dos de variantes (de autores no identificados).

Cerrando la cuarta división, encontramos algunas consideraciones generales, válidas para todo su sistema métrico: Nos dice Trissino que sólo ha ejemplificado con trímetros y dímetros (porque, como dijo en la segunda división, son las medidas habituales en la poesía italiana), pero la teoría podría adaptarse a otras medidas. Ha ejemplificado con trocaicos, pero cabría adaptar lo que ha dicho a los yámbicos. Y, cosa importantísima para nosotros, cosa que no habían hecho ni Dante ni Tempo, y Trissino es el primero en hacer: Presta atención al ritmo acentual de todo el poema. Afirma:

Estoy convencido de que de ningún modo se deban mezclar yámbicos con trocaicos, como hicieron los antiguos. Y ello porque, al ser absolutamente contrarios entre sí, dan diversa resonancia, y consecuentemente discordancia. Pero quien los quiera usar, podrá usarlos por sí mismo.

Apunta después algún posible desarrollo métrico: los trímetros "no serían quizá inútiles en las comedias, para quien los supiese usar". Disculpa la extensión de estas divisiones ${ }^{35}$ : "Porque, queriendo dar diligente conocimiento de ellas [las

DANTE las llama "cantos", pero comúnmente se llaman "capítulos".

35 Las cuatro primeras divisiones, en la edición príncipe, son $70 \mathrm{pp}$. recto/verso, por tanto 140 páginas. Y las dos últimas divisiones, numeradas de nuevo, serán 47 páginas, también recto/verso, por tanto 94 . Lo que hace un total, para la Poética completa, de 234 páginas. 
"rimas": los poemas, "y las cosas que pertenecen a ellas"], me he extendido demasiado." Y anuncia el contenido de las dos divisiones que ahora no publica: "Pero en los otros dos libros que quedan se tratará de la Tragedia, de la Comedia, del Heroico, y universalmente de todos los poemas, con la mayor diligencia y brevedad por nuestra parte que se podrá." Indica que no ha podido estampar actualmente esas dos divisiones,

pero si Dios quiere se estamparán. Entre tanto, tomaréis éstas, que tratan por extenso de las rimas y de muchas cosas bellas y recónditas que se relacionan con ellas.

\section{Conclusión}

Una Poética que comienza así: "Bellísima cosa es hacer el bien a las gentes...", con esa palabra, "bellísima", que revela la enorme sensibilidad estética de Trissino; y una Poética que termina en su primera parte publicada (1929) resumiendo Trissino su aportación así: he tratado de las "rimas", y de las "muchas cosas bellas y recónditas" que esconden las rimas (nuevamente apuntando a la belleza y al misterio descifrable de la Poesía), no cabe duda de que es obra, no solo de un erudito, sino también de un artista.

Además de esta extraordinaria sensibilidad, muchos son los méritos que avalan la teoría métrica de Trissino. En primer lugar -en línea con lo que acabamos de decir-, el gran acierto estético de los ejemplos literarios que clarifican y adornan su teoría: pertenecen, mayoritariamente, a Petrarca, y en segundo lugar a Dante. Entre ambos, cumbres de la literatura italiana, dan respuesta a una gran mayoría de las cuestiones métricas que plantea Trissino. Esto puede considerarse señal de gran admiración y entusiamo hacia ellos. Después, a mucha distancia, vienen otros nombres, bien próximos en el tiempo (Boccaccio, a quien nuestro autor también respeta), bien más alejados (poetas del "dolce stil novo", en particular Cino da Pistoia, al que cita con frecuencia gustosamente), o alejadísimos, como los poetas sículo-toscanos del Duecento (sobre todo el gran Guittone d'Arezzo). En contadas 
ocasiones se refiere a su propia obra, que, sin embargo, es muy notable poéticamente. En unos pocos casos, el ejemplo literario es flojo, pero cubre la estructura métrica que está exponiendo Trissino: no encontraría otro mejor. En resumen, el juicio estético de Trissino, privilegiando los grandes modelos, es impecable, y coincide con el juicio de la posteridad. Además, "los buenos autores" son la guía de la Poética, no sólo por su práctica sino también por su teoría (implícita): las formas que ellos han privilegiado son las que Trissino recoge y elabora. Contribuye nuestro autor así a fijar el canon poético italiano.

Podríamos decir que esta es una primera gran diferencia con Tempo. Éste aporta pésimos ejemplos poéticos, con gran pobreza de inspiración y con una total anarquía en el ritmo acentual de los versos, que raspan. Parecen salidos todos de la misma pluma -el estilo es el mismo-. Nunca cita el autor, pero podemos suponerlos de su invención, tal como harán después cantidad de metricistas en los siglos siguientes (p. ej, en la literatura española, el autor de La pícara Justina).

Pasando ahora a la teoría métrica de Trissino, centro de nuestro trabajo, creemos que su clave está en la gran cultura clásica del autor. Al igual que sucede en su literatura, que renueva la de su tiempo enlazándola (tragedia, comedia, poema heroico) con la grecolatina, así también en su teoría métrica. Detallemos sus principales aportes:

$1^{\mathrm{o}}$.- Descubrimiento y consideración de la función del acento en el verso. Con el modelo subyacente de la métrica clásica (sílabas largas y breves, pies), Trissino establece la importancia del ritmo del verso sobre la base de las sílabas átonas ("graves") y tónicas ("agudas"). Nacen así los modernos "pies", no cuantitativos sino acentuales, y surgen los versos yámbicos, trocaicos, etc.

$2^{\circ}$ - Establecimiento de un sistema para describir la longitud de los versos, no sobre la base del número de sílabas, sino sobre la base de los "metros" o "medidas" (reunión de pies): trímetros, dímetros, etc. Lo cual, unido 
a la estructura acentual de los pies, nos da también la melodía de los versos: trímetros trocaicos, dímetros yámbicos, etc. Trissino crea, así, un sistema racional descriptivo ${ }^{36}$.

$3^{\circ}$ - La descripción de los cinco tipos poemáticos vigentes en la época de Trissino (soneto, canción, balada, madrigal y tercetos encadenados) -en la cuarta división de la Poética-, a los que hay que añadir el endecasílabo suelto -en la sexta división-, es sencillamente magnífica e insuperable por su exactitud, honradez intelectual y sensibilidad. Puesto que, además, la canción incluye la sextina, podemos decir que Trissino plasma conceptualmente siete tipos poemáticos con sus variantes. Dos rasgos caracterizan el estudio trissiniano: método sistemático, y atención al uso, especialmente al uso de los buenos autores.

$4^{\mathrm{o}}$ - Este programa tan completo, en una época en que la teoría métrica carecía de tradición de estudio, necesitaba poner en pie todo un edificio terminológico. Trissino lo hace, con notable fortuna, y da cuenta así de la complejidad, pero también de la lógica, de la ciencia que hoy llamamos Métrica.

Para concluir, señalemos algunos juicios de estudiosos italianos. Primero el de Francesco Bausi y Mario Martelli:

[...] Trissino, la cui Poetica, di stretta osservanza aristotelica, è certo l'opera più agguerrita e completa del secolo sul piano della teoria e dell'analisi metrica (proprio il Trissino, non a caso, riportò alla luce e fece conoscere il De vulgari eloquentia dantesco $)^{37}$.

Y por último el de Guglielmo Gorni, que enlaza con la cita inicial:

Nella "quarta divisione" della Poetica (1529) di Gian Giorgio

36 Como señala PAZZAGLIA, Mario (Manuale di Metrica italiana, cit., pp. 3940), el Medioevo había estado atento al número de sílabas y a la rima, pero la idea acentuativa del verso italiano surge con la Poética de TRISSINO.

37 BAUSI, Francesco, y MARTELLI, Mario: La metrica italiana, cit., p. 174. 
Trissino, il più sottile ingegno metrico del Rinascimento, si fa luce una portentosa coscienza tecnica del testo poetico: un'esperienza cosí solida e sicura di metrica volgare non si era piú vista dal tempo del De vulgari eloquentia, opera riscoperta, e non è un caso, proprio dal Trissino [...] (p. 441).

Il modello teorico e storiografico del Trissino, che fu anche poeta generosamente innovativo, resterà, per $i$ fatti metrici, insuperato $(p$. $442)^{38}$.

\section{Bibliografía}

ALIGHIERI, Dante. Véase DANTE.

ANDREWS, Richard: "Premessa" a la edición de Tempo, p. I. Véase TEMPO. Atti del Convegno di studi su Giangiorgio Trissino. A cura di Neri Pozza. Vicenza: Accademia Olimpica, 1980.

BARBIERI, Franco: "Giangiorgio Trissino e Andrea Palladio", en Atti del Convegno di studi su Giangiorgio Trissino. A cura di Neri Pozza. Vicenza: Accademia Olimpica, 1980, pp. 191-211.

BAUSI, Francesco y MARTELLI, Mario: La metrica italiana. Teoria e storia. Firenze: Casa Editrice Le Lettere, 1993.

BELTRAMI, Pietro G.: La metrica italiana. Bologna: Il Mulino, 2a ed. 1994.

BEMBO, Pietro: Prose della volgar lingua (1525). http://www.classicitaliani.it/ bembo/bembo03.htm (01/09/2010)

CARAMUEL, Juan: Primus Calamus, 2 t. (1663 y 1665). Trad. esp.: Primer Cálamo, tomo II: Rítmica. Ed. y estudio de Isabel Paraíso. Valladolid: Universidad de Valladolid, 2007, $565 \mathrm{pp}$.

DANTE (Alighieri): De Vulgari Eloquentia (c. 1304; publ. por Trissino en 1529). Milano: Signorelli, 1988.

DÍAZ RENGIFO. Véase RENGIFO.

DIETRICK, Déborah: "De consonancia a rima", en Antonio Álvarez Tejedor y AA. VV: Lengua viva. Estudios ofrecidos a César Hernández Alonso. Valladolid: Universidad de Valladolid, 2008, pp. 1055-1073.

GORNI, Guglielmo: "Le forme del testo poetico", en Alberto A. Rosa (dir.): Letteratura italiana, vol. III, Le forme del testo. 1. Teoria e poesia. Torino: Giulio Einaudi Editore, 1984, pp. 16 y 439-518.

MARTELLI, Mario: "Le forme poetiche italiane dal Cinquecento ai nostri giorni”, en Alberto A. Rosa (dir.): Letteratura italiana, vol. III, Le forme del testo. 1. Teoria e poesia. Torino: Giulio Einaudi editore, 1984, pp. 518-620.

MORSOLIN, Bernardo: Giangiorgio Trissino. Monografia d'un gentiluomo letterato nel secolo XVI. Seconda edizione corretta e ampliata. Firenza: Successori Le Monnier, 1894, 492 pp.

38 GORNI, Guglielmo: "Le forme del testo poetico", en Alberto A. Rosa (dir.): Letteratura italiana, vol. III, Le forme del testo. 1. Teoria e poesia. Torino: Giulio Einaudi Editore, 1984, pp. 441-442. 
PAGLIERANI, Franco (ed.): La Sofonisba di Giangiorgio Trissino, con note di Torquato Tasso. Bologna: Presso Gaetano Romagnoli, 1884, 40 pp.

PARAÍSO, Isabel: "Fundación del canon métrico: El Arte Poética Española, de Juan Díaz Rengifo", en Isabel Paraíso (coord.): Retóricas y Poéticas Españolas, siglos XVI-XIX. Valladolid: Univ. de Valladolid, 2000, pp. 47-93.

- La Métrica española en su contexto románico. Madrid: Arco/Libros, 2000.

PAZZAGLIA, Mario: Manuale di Metrica italiana. Firenze: Sansoni, 1990.

PERNICONE, Vincenzo: "Storia e svolgimento della metrica", en M. Fubini, G. Getto, B. Migliorini, A. Chiari, V. Pernicone: Tecnica e teoria lettteraria. Milano: Carlo Marzorati Editore, 1951, pp. 297-349.

RENGIFO (Juan Díaz Rengifo): Arte Poética Española. Salamanca: Miguel Serrano de Vargas, 1592. Ed. más asequible: Facsímil de la 2a ed. (Madrid: Juan de la Cuesta, 1606). Valladolid: Editorial Maxtor, 2007.

RUSCELLI, Girolamo: Rimario del Signor Girolamo Ruscelli colla dichiarazione, colle regole e col giudizio, per saper convenevolmente usare, o schifare le voci nell'esser, loro cosi nelle Prose, come ne'Versi: Premessovi il Trattato Del modo di comporre in Versi nella Lingua Italiana, del medesimo Autore. [1559]. Nuova edizione, Venezia: dai Torchi di Giuseppe Molinari, 1827.

TEMPO, Antonio da: Summa Artis Rithimici Vulgaris Dictaminis (1332; nueva publ. Venezia, 1509). Edizione critica a cura di Richard Andrews. Bologna: Commisione per i Testi di Lingua, 1977.

TRISSINO, Giangiorgio: La Poetica [1529 y 1562]. Ed. facsímil. München: Fink Verlag, Poetiken des Cinquecento, 1969.

- La Sofonisba. (Véase PAGLIERANI).

-(http://en.wikipedia.org/wiki/Gian_Giorgio_Trissino, 04/05/2009).

VEGA, María José: "La Poética hermogénica renācentista: Giovan Giorgio Trissino". Rev. Castilla (Universidad de Valladolid), 1991, 16, pp. 169-188.

WEINBERG, Bernard: A History of Literary Criticism in the Italian Renaissance, 2 vols. The University of Chicago Press, 1961. En vol. I, pp. 368-70, y en vol. II, $750-55$ y $1070-71$. 